\title{
COMPACTIFYING INFINITE GROUP ACTIONS
}

\author{
Ian Hambleton and Erik K. Pedersen
}

June 16, 1999

\begin{abstract}
Conditions are given under which discrete co-compact group actions on $S^{n} \times \mathbf{R}^{k}$ extend to actions on $S^{n+k}$.
\end{abstract}

\section{INTRODUCTION}

In a previous paper [6] we studied free, properly discontinuous co-compact actions of certain infinite discrete groups $\Gamma$ on $S^{n} \times \mathbf{R}^{k}$. The goal was to find restrictions on the finite subgroups of $\Gamma$ by showing that the action $\left(S^{n} \times \mathbf{R}^{k}, \Gamma\right)$ restricted to any finite subgroup $G \subset \Gamma$ could be completed to an action of $G$ on the sphere $S^{n+k}$, free on the complement of a standardly embedded $G$-invariant subsphere $S^{k-1}$.

In this paper we consider the problem of completing the $\Gamma$ action. The examples we obtain give many new actions of discrete groups on spheres $S^{n+k}$ with limit sets contained in a $\Gamma$-invariant subsphere $S^{k-1}$. There is an extensive literature on this subject (see [9]) arising from the classical theory of Kleinian groups.

To state our main criterion for compactifying $\Gamma$ actions on $S^{n} \times \mathbf{R}^{k}$ we will use the definitions of Lipschitz homotopy equivalence from Section 1 (introduced in [7, $\S 11]$ ) and of an action which is eventually small at infinity given in Section 3. This material owes a lot to the foundational work of M. Gromov (see for example [4]). Recall that a torsion-free group $\Gamma_{0}$ has a classifying space $B \Gamma_{0}$ with contractible universal covering space $E \Gamma_{0}$ on which $\Gamma_{0}$ acts freely and properly discontinuously. We always assume that $B \Gamma_{0}$ is compact and give $E \Gamma_{0}$ the metric induced from a metric on $B \Gamma_{0}$, so that $\Gamma_{0}$ acts by isometries on $E \Gamma_{0}$.

Definition 1.1. A group $\Gamma$ is said to be eventually $(\alpha, k)$-euclidean if $v c d(\Gamma)<\infty$ and it has a torsion-free normal subgroup $\Gamma_{0}$ of finite index with $B \Gamma_{0}$ compact, such that

(i) $\Gamma$ acts by isometries on $E \Gamma_{0}$ extending the $\Gamma_{0}$ action, properly discontinuously, co-compactly and with finite isotropy,

(ii) $E \Gamma_{0}$ is Lipschitz homotopy equivalent to $\mathbf{R}^{k}$,

(iii) $E \Gamma_{0}$ has a $\Gamma$-equivariant compactification $\left(\overline{E \Gamma}_{0}, \Gamma\right)=\left(D^{k}, \Gamma\right)$ where the action is eventually small at infinity, and

(iv) the action of $\Gamma$ restricted to the boundary of $D^{k}$ is given by a homomorphism $\alpha: \Gamma \rightarrow \operatorname{Homeo}\left(S^{k-1}\right)$.

The authors wish to thank the Max Planck Institut für Mathematik in Bonn for its hospitality and support. The first author was partially supported by NSERC and second author by NSF grant DMS-9104026. 
Theorem A. Let $\Gamma$ be a group which is eventually $(\alpha, k)$-euclidean. If $\Gamma$ acts freely, properly discontinuously and co-compactly on $S^{n} \times \mathbf{R}^{k}$ then there exists a compactification $\left(S^{n+k}, \Gamma\right)$ such that

(i) there is a $\Gamma$-invariant linear subsphere $S^{k-1}$ in $S^{n} \times \mathbf{R}^{k}$,

(ii) the action on $S^{n+k}-S^{k-1}=S^{n} \times \mathbf{R}^{k}$ is topologically conjugate to the given action, and

(iii) the $\Gamma$ action on $S^{k-1}$ is given by $\alpha$.

These conditions hold if $\Gamma$ is a group of isometries of a complete Riemannian manifold with non-positive curvature. We therefore obtain examples of the form $\Gamma=\mathbf{Z}^{k} \rtimes D$ or $\Gamma=\Delta \rtimes D$, where $D$ is a finite group acting freely on a sphere and $\Delta$ is the fundamental group of a hyperbolic manifold. More examples arise from the existence results of $[6,8.3]$.

\section{Lipschitz Homotopy Equivalence}

We work in the category of proper metric spaces and proper maps. Recall that a metric space is proper if all closed metric balls are compact. A proper map $f: X \rightarrow Y$ between metric spaces is proper if the inverse image of any bounded set is bounded. A map $f: X \rightarrow Y$ is eventually Lipschitz if there are constants $K>0$, $L \geq 0$ such that $d\left(f(x), f\left(x^{\prime}\right)\right) \leq K d\left(x, x^{\prime}\right)+L$ for all $x, x^{\prime} \in X$. If $L=0$ the map is called Lipschitz.

Definition 2.1. Let $f_{0}, f_{1}: X \rightarrow Y$ be proper, Lipschitz maps between proper metric spaces. They are called Lipschitz homotopy equivalent (written $f_{0} \simeq_{\text {Lip }} f_{1}$ if there exists a proper Lipschitz map $H: X \times \mathbf{R} \rightarrow Y \times \mathbf{R}$ of the form $H(x, t)=$ $\left(h_{t}(x), t\right)$ and a continuous function $\phi: X \rightarrow[0, \infty)$ such that

(i) $h_{t}(x)=f_{1}(x)$ if $t \geq \phi(x)$, and

(ii) $h_{t}(x)=f_{0}(x)$ if $t \leq 0$.

We remark that Lipschitz homotopy equivalence is a reflexive relation, but it is not clear whether it is symmetric or transitive.

Example 2.2. Let $X=Y=O(K)$ with $f_{0}(t x)=2 t x, f_{1}(t x)=t x$. Then $f_{1} \simeq_{L i p}$ $f_{2}$ using the map $\phi(t x)=2 t$.

Remark 2.3. The inclusion map of the subspace $N=\{(x, \phi(x)) \mid x \in X\}$ is not necessarily a Lipschitz map into $X \times \mathbf{R}$, but $N$ is homeomorphic to $X$.

Definition 2.4. Two proper metric spaces $X, Y$ are Lipschitz homotopy equivalent (written $X \simeq_{\text {Lip }} Y$ ) if there exist proper Lipschitz maps $f: X \rightarrow Y$ and $g: Y \rightarrow X$ such that $g \circ f \simeq_{L i p} i d_{X}$ and $f \circ g \simeq_{L i p} i d_{Y}$.

The analogous definition using eventually Lipschitz maps will be called eventually Lipschitz homotopy equivalent. For example, two metric spaces $X$ and $Y$ which are quasi-isometric are eventually Lipschitz homotopy equivalent. A special case to keep in mind is any subgroup $\Gamma_{0} \subset \Gamma$ of finite index, where $\Gamma$ is a finitely generated discrete group with the word metric. The inclusion map is a quasi-isometry, hence $\Gamma_{0}$ and $\Gamma$ are eventually Lipschitz homotopy equivalent.

Example 2.5. The subspace of $\mathbf{R}^{3}$ given by the union of the half cylinder

$$
\left\{(x, y, z) \in \mathbf{R}^{3} \mid x^{2}+y^{2}=1, z \geq 0\right\}
$$


together with its circular base $\left\{x^{2}+y^{2} \leq 1, z=0\right\}$ is homeomorphic to $\mathbf{R}^{2}$, but not Lipschitz homotopy equivalent to $\mathbf{R}^{2}$.

Example 2.6. Let $M$ be a complete simply-connected Riemannian $k$-manifold of non-positive curvature. Then $M \simeq_{L i p} \mathbf{R}^{k}$ using the exponential map $\exp _{x}: T_{x} M \rightarrow$ $M$ and its inverse, the logarithm map. The logarithm map is Lipschitz but the exponential map must be modified by composing with a radial contraction to make it Lipschitz.

Theorem 2.7. Suppose that $f_{0}$ and $f_{1}$ are proper Lipschitz maps from $X \rightarrow Y$ and $f_{0} \simeq_{\text {Lip }} f_{1}$. Then the induced functors $\left(f_{0}\right)_{*}$ and $\left(f_{1}\right)_{*}$ from $\mathcal{C}_{X}(R) \rightarrow \mathcal{C}_{Y}(R)$ give the same maps on $K$-theory and $L$-theory.

Proof. We include a sketch of the proof (following the argument in [7, 11.3]). Consider the following subspaces of $X \times \mathbf{R}$ : let $M=\{(x, 0) \mid x \in X\}, N=$ $\{(x, \phi(x)) \mid x \in X\}$ and $W=\{(x, t) \mid 0 \leq t \leq \phi(x)\}$. We have the inclusion maps $\iota_{0}: M \rightarrow W$ and $\iota_{1}: N \rightarrow W$. By excision, both $\iota_{0}$ and $\iota_{1}$ induce isomorphisms on bounded $K$ or $L$ theory with appropriate decorations.

Projection $(x, t) \mapsto(x, 0)$ gives maps $p_{1}: N \rightarrow M$ and $p_{W}: W \rightarrow M$, with $p_{W} \circ$ $\iota_{1}=p_{1}$ and $p_{W} \circ \iota_{0}=i d_{M}$. By construction, $H \circ \iota_{1}=f_{1} \circ p_{1}$ and $H \circ \iota_{0}=f_{0}$ Therefore

$$
\begin{aligned}
H_{*} \circ\left(\iota_{1}\right)_{*} & =\left(f_{1}\right)_{*} \circ\left(p_{1}\right)_{*} \\
& =\left(f_{1}\right)_{*} \circ\left(p_{W}\right)_{*} \circ\left(\iota_{1}\right)_{*}
\end{aligned}
$$

But $\left(\iota_{1}\right)_{*}$ is an isomorphism, so $H_{*}=\left(f_{1}\right)_{*} \circ\left(p_{W}\right)_{*}$. Now

$$
\left(f_{1}\right)_{*}=\left(f_{1}\right)_{*} \circ\left(p_{W}\right)_{*} \circ\left(\iota_{0}\right)_{*}=H_{*} \circ\left(\iota_{0}\right)_{*}=\left(f_{0}\right)_{*}
$$

Corollary 2.8. Let $\Gamma$ be eventually $(\alpha, k)$-euclidean. Then the bounded TOP structure set of $S^{n} \times E \Gamma_{0}$, bounded with respect to the second factor projection $p: S^{n} \times E \Gamma_{0} \rightarrow E \Gamma_{0}$, contains only the base point if $n+k \geq 5$.

Proof. We compare the bounded surgery exact sequences [3] for $p: S^{n} \times E \Gamma_{0} \rightarrow E \Gamma_{0}$ and $\ell \circ p: S^{n} \times E \Gamma_{0} \rightarrow \mathbf{R}^{k}$, by composing with a Lipschitz homotopy equivalence $\ell: E \Gamma_{0} \rightarrow \mathbf{R}^{k}$ at the control space level. This gives a well-defined map of surgery exact sequences, inducing an isomorphism on the normal invariant and $L$-group terms. Therefore the bounded structure set of $p: S^{n} \times E \Gamma_{0} \rightarrow E \Gamma_{0}$ has a bijection to the bounded structure set of $\ell \circ p: S^{n} \times E \Gamma_{0} \rightarrow \mathbf{R}^{k}$. By assumption, there is a homeomorphism $E \Gamma_{0}=\operatorname{int}\left(D^{k}\right)$ and we compose with a radial identifcation $\operatorname{int}\left(D^{k}\right) \approx \mathbf{R}^{k}$ to get a homeomorphism $h: E \Gamma_{0} \rightarrow \mathbf{R}^{k}$. The map $1 \times h: S^{n} \times E \Gamma_{0} \rightarrow$ $S^{n} \times \mathbf{R}^{k}$ gives a bijection of bounded structure sets, and we note that the bounded structure set of $p_{2}: S^{n} \times \mathbf{R}^{k} \rightarrow \mathbf{R}^{k}$ contains only the base point.

\section{Control at Infinity}

Let $X$ be a topological space.

Definition 3.1. A topological action $(X, \Gamma)$ is continuously controlled at a $\Gamma$ invariant subset $A \subset X$ provided that: for all compact subsets $K \subset X-A$, and 
for each neighbourhood $U$ of $x \in A$, there exists a neighbourhood $V \subset U$ of $x$ such that whenever $\gamma \cdot K \cap V \neq \emptyset$, for some $\gamma \in \Gamma$, it follows that $\gamma \cdot K \subset U$.

Our main application is to the compactifications of classifying spaces for discrete groups. For our purposes, a compactification of $E \Gamma_{0}$ is a compact, contractible topological space $\overline{E \Gamma}_{0}$ containing $E \Gamma_{0}$ as a dense open subset. The frontier of $\overline{E \Gamma}_{0}$ is $\overline{E \Gamma}_{0}-E \Gamma_{0}$, denoted by $\partial \overline{E \Gamma}_{0}$.

Definition 3.2. Let $\Gamma_{0} \subset \Gamma$ be a torsion-free subgroup and suppose that $\Gamma$ acts by isometries on $E \Gamma_{0}$. If $\left(E \Gamma_{0}, \Gamma\right)$ has a $\Gamma$-equivariant compactification $\left(\overline{E \Gamma}_{0}, \Gamma\right)$, then we say that the action is eventually small at infinity if $\left(\overline{E \Gamma}_{0}, \Gamma\right)$ is continuously controlled at the frontier $\partial \overline{E \Gamma}_{0}$.

There is another control condition at infinity using the metric on $E \Gamma_{0}$.

Definition 3.3. Let $\Gamma$ act properly discontinuously and co-compactly on $E \Gamma_{0}$, and suppose that $\left(E \Gamma_{0}, \Gamma\right)$ has a $\Gamma$-equivariant compactification $\left(\overline{E \Gamma}_{0}, \Gamma\right)$. The group $\Gamma$ is small at infinity provided that: for all metric balls $B(k) \subset E \Gamma_{0}$ and for each $x \in \overline{E \Gamma}_{0}-E \Gamma_{0}$ and neighbourhood $U$ of $x$ in $\overline{E \Gamma}_{0}$, there exists a neighbourhood $V \subset U$ of $x$ such that whenever $B(k) \cap V \neq \emptyset$, it follows that $B(k) \subset U$.

Note that since $E \Gamma_{0}$ is a proper metric space and $\Gamma$ acts by isometries, a group $\Gamma$ which is small at infinity has the given action $\left(\overline{E \Gamma}_{0}, \Gamma\right)$ eventually small at infinity. In our situation, these two conditions are actually equivalent.

Proposition 3.4. Suppose that the action $\left(\overline{E \Gamma}_{0}, \Gamma\right)$ is eventually small at infinity. Then the group $\Gamma$ is small at infinity.

Proof. Since the action $\left(E \Gamma_{0}, \Gamma_{0}\right)$ is co-compact, we can choose a point $x_{0} \in E \Gamma_{0}$ and $k$ so large that any metric ball $B(k)$ contains a point of the form $\gamma \cdot x_{0}$ for some $\gamma \in \Gamma$. Given $x \in \overline{E \Gamma}_{0}-E \Gamma_{0}$, and a neighbourhood $U$ of $x$ in $\overline{E \Gamma}_{0}$, there exists a neigbourhood $V \subset U$ such that

$$
\gamma \cdot B\left(x_{0}, 2 k\right) \cap V \neq \emptyset \quad \text { implies } \quad \gamma \cdot B\left(x_{0}, 2 k\right) \subset U .
$$

This is just the condition that the $\Gamma$ action is eventually small at infinity.

Now suppose that $B(k) \cap V \neq \emptyset$ for some metric ball $B(k)$. Then $\gamma \cdot x_{0} \in B(k)$ for some $\gamma \in \Gamma$ and it follows that

$$
B(k) \subset B\left(\gamma \cdot x_{0}, 2 k\right)=\gamma \cdot B\left(x_{0}, 2 k\right)
$$

where the last equality follows since $\Gamma$ acts by isometries on $E \Gamma_{0}$. But now we conclude from (3.5) that $B(k) \subset U$.

\section{Almost Equivariant Projections}

We suppose now that $\Gamma$ is eventually $(\alpha, k)$-euclidean. If $\Gamma$ acts freely, properly discontinuously and co-compactly on $S^{n} \times \mathbf{R}^{k}$ then we have a compact manifold $M=S^{n} \times \mathbf{R}^{k} / \Gamma_{0}$ and a classifying map $M \rightarrow B \Gamma_{0}$. Up to homotopy this is a spherical fibration with fibre $S^{n}$, so we can replace it by a block fibration $\bar{M} \rightarrow$ $B \Gamma_{0}$ with $\bar{M}$ still compact and homotopy equivalent to $M$. The universal cover $\widehat{M}$ of $\bar{M}$ is a block fibration $q: \widehat{M} \rightarrow E \Gamma_{0}$ over $E \Gamma_{0}$, which is contractible, so it is block and hence boundedly homotopy equivalent to the trivial block fibration 
$S^{n} \times E \Gamma_{0} \rightarrow E \Gamma_{0}$. Here we are using the blocked structures with respect to a $\Gamma_{0}$-equivariant triangulation of $E \Gamma_{0}$, so the simplices have a bounded diameter.

Now if $\widetilde{M}$ denotes the universal covering of $M$, we obtain a bounded homotopy equivalence $f: \widetilde{M} \rightarrow S^{n} \times E \Gamma_{0}$, bounded with respect to the second factor projection $p: S^{n} \times E \Gamma_{0} \rightarrow E \Gamma_{0}$.

Definition 4.1. Let $X$ be a $\Gamma$ space and $Z$ be a metric space on which $\Gamma$ acts by isometries. Given a $\Gamma$ equivariant map $p: X \rightarrow Z$, we say that $p$ is almost equivariant if there exists a constant $k>0$ such that $d(\gamma \cdot p(x), p(\gamma \cdot x))<k$ for all $x \in X$. If the bound $k$ is independent of $\gamma \in \Gamma$, we say that $p$ is uniformly almost equivariant

Lemma 4.2. The map $p f: \widetilde{M} \rightarrow E \Gamma_{0}$ is uniformly almost $\Gamma_{0}$-equivariant.

Proof. The map $f$ is the composite of a $\Gamma_{0}$-equivariant bounded homotopy equivalence $\widetilde{M} \rightarrow \widehat{M}$ (covering the homotopy equivalence $M \rightarrow \bar{M}$ ) and a bounded homotopy equivalence $j: \widehat{M} \rightarrow S^{n} \times E \Gamma_{0}$. Since $p \circ j$ is bounded homotopy equivalent to $q$, the distance $d(p j(\gamma \cdot x), \gamma \cdot p j(x))$ differs by a bounded amount independent of $\gamma$ from $d(q(\gamma \cdot x), \gamma \cdot q(x))$. But $q$ is $\Gamma_{0}$-equivariant so this last distance is zero.

Since $\Gamma$ is eventually $(\alpha, k)$-euclidean, the bounded structure set of $S^{n} \times E \Gamma_{0} \rightarrow$ $E \Gamma_{0}$ contains just one element (represented by the identity map, see Corollary $2.8)$. Therefore there is a bounded homotopy from $f$ to a homeomorphism $h: \widetilde{M} \rightarrow$ $S^{n} \times E \Gamma_{0}$. On $\widetilde{M}$ we have the given free $\Gamma$ action, so we can consider the conjugate $\Gamma$ action by $h$ on $S^{n} \times E \Gamma_{0}$.

Lemma 4.3. The second factor projection map $p: S^{n} \times E \Gamma_{0} \rightarrow E \Gamma_{0}$ is uniformly almost equivariant with respect to the conjugated $\Gamma$ action restricted to $\Gamma_{0}$.

Proof. We consider the quantity

$$
d\left(p h z h^{-1} x, z p x\right) \leq d\left(p h z h^{-1} x, p f\left(z h^{-1} x\right)\right)+d\left(p f\left(z h^{-1} x\right), z p x\right)
$$

where $z \in \Gamma_{0}$ and $x \in S^{n} \times E \Gamma_{0}$. The first term is bounded since $f$ is boundedly homotopic to $h$. The second term is a bounded distance from $d\left(z p f\left(h^{-1} x\right), z p x\right)$ since $p f$ is uniformly almost $\Gamma_{0}$-equivariant by (4.2). Since $\Gamma_{0}$ acts by isometries on $E \Gamma_{0}$, this last term is equal to $d\left(p f\left(h^{-1} x\right), p x\right)$ which is bounded since $f$ is boundedly homotopic to $h$.

The main result of this section is that this almost equivariance property holds for the $\Gamma$ action as well.

Theorem 4.4. The second factor projection map $p: S^{n} \times E \Gamma_{0} \rightarrow E \Gamma_{0}$ is uniformly almost equivariant with respect to the conjugated $\Gamma$ action.

Proof. Choose a compact fundamental domain $U$ for the $\Gamma_{0}$ action on $S^{n} \times E \Gamma_{0}$, so that the sets $\left\{z \cdot U \mid z \in \Gamma_{0}\right\}$ cover $S^{n} \times E \Gamma_{0}$. Let $\left\{g_{i} \mid 1 \leq i \leq m\right\}$ be a set of coset representatives for $\Gamma / G_{0}$, and let

$$
U_{1}=\bigcup_{1 \leq i \leq m} g_{i} \cdot U
$$

Since $U$ is compact, the set

$$
\bar{U}_{1}=\bigcup_{1 \leq i \leq m} g_{i} \cdot p\left(U_{1}\right) \subset E \Gamma_{0}
$$


has finite diameter $d$.

For $x \in S^{n} \times E \Gamma_{0}$, write $x=z u$ where $z \in \Gamma_{0}$ and $u \in U$, and let $g=g_{i}$ for some $i, 1 \leq i \leq m$. Then

$$
\begin{aligned}
d(p g x, g p x) & =d(p g z u, g p z u) \\
& \leq d\left(p g z u, g z g^{-1} p g u\right)+d\left(g z g^{-1} p g u, g z p u\right)+d(g z p u, g p z u)
\end{aligned}
$$

The first term is

$$
d\left(p g z u, g z g^{-1} p g u\right)=d\left(p g z g^{-1} g u, g z g^{-1} p g u\right)<k
$$

since $g z g^{-1} \in \Gamma_{0}$ by (4.3). This is the place where we need uniform almost equivariance for the map $p$ with respect to the $\Gamma_{0}$ action. The second term is

$$
d\left(g z g^{-1} p g u, g z p u\right)=d\left(g z g^{-1} p g u, g z g^{-1} g p u\right)=d(p g u, g p u)<d
$$

since $\Gamma_{0}$ acts by isometries on $E \Gamma_{0}$, and both $p(g u)$ and $g p(u)$ are in $\bar{U}_{1}$ which has diameter $d$. Finally, the third term

$$
d(g z p u, g p z u)=d(z p u, p z u)<k
$$

since $\Gamma$ acts by isometries and the projection $p$ is $\Gamma_{0}$-almost equivariant. From these estimates we get

$$
d(p \gamma x, \gamma p x)<3 k+d
$$

for all $\gamma \in \Gamma$ and all $x \in S^{n} \times E \Gamma_{0}$ so the projection $p$ is uniformly almost equivariant.

\section{The Proof of Theorem A}

We assume that the group $\Gamma$ is $(\alpha, k)$-euclidean and acts freely, properly discontinuously, and co-compactly on $S^{n} \times \mathbf{R}^{k}$. The results of the previous section say that $\left(S^{n} \times \mathbf{R}^{k}, \Gamma\right)$ is $\Gamma$ equivariantly homeomorphic to an action $\left(S^{n} \times E \Gamma_{0}, \Gamma\right)$ which has the additional property that the second factor projection $p: S^{n} \times E \Gamma_{0} \rightarrow E \Gamma_{0}$ is uniformly almost equivariant.

Proposition 5.1. Suppose that $\Gamma$ is small at infinity. Then for each $x_{0} \in \partial \bar{E}_{0}$, and each neighbourhood $U$ of $x_{0}$, there exists a neighbourhood $V \subset U$ of $x_{0}$ such that $p(x) \in V$, for $x \in S^{n} \times E \Gamma_{0}$, implies that $\gamma^{-1} \cdot p(\gamma \cdot x) \in U$ for all $\gamma \in \Gamma$.

Proof. Let $k$ denote a uniform bound for the $\Gamma$-almost equivariance of the map $p: S^{n} \times E \Gamma_{0} \rightarrow E \Gamma_{0}$, with respect to the action on $S^{n} \times E \Gamma_{0}$ described above. For $U$ and $x_{0}$ given, according to Definition 3.3 there exists a neigbourhood $V \subset U$ of $x_{0}$ such that $B(k) \cap V \neq \emptyset$ implies that $B(k) \subset U$. Now if $p(x) \in V$, for some $x \in S^{n} \times E \Gamma_{0}$, then we apply this to the $k$-ball centered at $p(x)$, and obtain $B(p(x), k) \subset U$. But

$$
d\left(\gamma^{-1} \cdot p(\gamma \cdot x), p(x)\right)=d\left(\gamma^{-1} \cdot p(\gamma \cdot x), p\left(\gamma^{-1} \gamma \cdot x\right)\right)<k
$$

so that $\gamma^{-1} \cdot p(\gamma \cdot x) \in B(p(x), k)$ implies $\gamma^{-1} \cdot p(\gamma \cdot x) \in U$.

Corollary 5.2. The action $\left(S^{n} \times \mathbf{R}^{k}, \Gamma\right)$ extends to a topological $\Gamma$-action on $S^{n+k}=S^{n} \times \mathbf{R}^{k} \cup S^{k-1}$ where the action on $S^{k-1}$ is given by the action $\alpha$ on $\partial \overline{E \Gamma}_{0}=\partial D^{k}=S^{k-1}$.

Proof. The preceeding result shows that for each $\gamma \in \Gamma$, the given action of $\gamma$ on $S^{n} \times \mathbf{R}^{k}$ together with the action given by $\alpha(\gamma)$ on $S^{k-1}$ fit together to give a homeomorphism of $S^{n+k}$. 


\section{Future Developments}

Some of the definitions given earlier suggest questions for further study. Probably the formulations below are too naive.

Question 6.1. Let $(X, d)$ be a metric space homeomorphic to $\mathbf{R}^{k}$, and suppose that $(X, d)$ is also Lipschitz homotopy equivalent to $\mathbf{R}^{k}$. Does a finite group acting by isometries on $X$ necessarily have a fixed point ?

We remark that there exist smooth fixed-point free actions of finite cyclic groups on $\mathbf{R}^{k}$ (see [8]), but these actions do not preserve the standard metric on $\mathbf{R}^{k}$.

Question 6.2. Suppose that $\left(\overline{E \Gamma}_{1}, \Gamma_{1}\right)$ and $\left(\overline{E \Gamma}_{2}, \Gamma_{2}\right)$ are actions which are eventually small at infinity. Does there exist an equivariant compactification of $E \Gamma_{1} \times E \Gamma_{2}$ which is eventually small at infinity?

An action of a discrete group is uniformly continuous if the usual $\epsilon-\delta$ continuity condition for each group element $\gamma \in \Gamma$ allows a $\delta$ depending only on $\epsilon$ (and not on the group element $\gamma$ ).

Question 6.3. Suppose that $\Gamma_{0} \subset \Gamma$ is a torsion-free normal subgroup of finite index and the action on $\left(\overline{E \Gamma}_{0}, \Gamma\right)$ is eventually small at infinity. Is the $\Gamma$-action on $\overline{E \Gamma}_{0}$ uniformly continuous?

We would certainly like to relax some of our assumptions on the discrete group $\Gamma$ in order to include more of the interesting classes of groups already appearing in the literature (see [1], [4]). As above, we let $\Gamma_{0} \subset \Gamma$ be a torsion-free normal subgroup of finite index. Suppose that $\left(\bar{E}_{0}, \Gamma\right)$ is an equivariant compactification of $E \Gamma_{0}$ with $\Gamma$-action eventually small at infinity. We will assume that there exists an embedding of $\overline{E \Gamma}_{0}$ in $S^{n+k}$ as a neighbourhood retract. Furthermore, we want to assume that $E \Gamma_{0}$ is compactified by a $Z$-set. In other words, $\overline{E \Gamma}_{0}$ comes equipped with a homotopy

$$
h_{t}: \overline{E \Gamma}_{0} \times[0,1] \rightarrow \overline{E \Gamma}_{0}
$$

such that $h_{0}=i d$, and the image $h_{t}(x) \notin \partial \overline{E \Gamma}_{0}$ for $t>0$. Using the retract and this homotopy, we can define a proper map $q: S^{n+k}-\partial \overline{E \Gamma}_{0} \rightarrow E \Gamma_{0}$ by the formula $h_{t}(r(x))$ where

$$
t=\frac{d\left(x, \overline{E \Gamma}_{0}\right)}{1+d\left(x, \overline{E \Gamma}_{0}\right)}
$$

and $r$ is the retract. We can now ask if the bounded structure set of

$$
q: S^{n+k}-\partial \overline{E \Gamma}_{0} \rightarrow E \Gamma_{0}
$$

contains just one element for $n+k \geq 5$. This is true for the bounded structure set of $S^{n+k}-K$ bounded over the open cone $O(K)$, provided that $K$ is a finite simplicial complex (see our paper [5,3.2]). Extending our techniques to handle complements of $\partial \overline{E \Gamma}_{0}$ instead of a finite complex $K$ looks like an interesting project, with other possible applications.

If $\Gamma$ acts freely, properly discontinuously, and co-compactly on $S^{n+k}-\partial \overline{E \Gamma}_{0}$ then all the additional information we need to extend the action to $S^{n+k}$ is the analogue of Corollary 2.8. The bounded homotopy type of the complement of the frontier (if $n \geq 2$ ) is again just given by the second factor projection $p: S^{n} \times E \Gamma_{0} \rightarrow E \Gamma_{0}$. This 
follows by the same arguments given at the beginning of Section 4, provided that $S^{n+k}-\partial \overline{E \Gamma}_{0}$ is homotopy equivalent to $S^{n}$. Since $\overline{E \Gamma}_{0}$ is contractible, this will follow for example if $\left(\overline{E \Gamma}_{0}, \partial \overline{E \Gamma}_{0}\right)$ is a $k$-dimensional Poincaré pair. In this situation, we need an affirmative answer from surgery theory to the following question.

Question 6.4. Suppose that $E \Gamma_{0}$ is a topological manifold. Does the bounded structure set of $S^{n} \times E \Gamma_{0} \rightarrow E \Gamma_{0}$ contain only the base point ? Equivalently, is every bounded homotopy equivalence $W \rightarrow S^{n} \times E \Gamma_{0}$, bounded with respect to the second factor projection $p: S^{n} \times E \Gamma_{0} \rightarrow E \Gamma_{0}$, boundedly homotopic to a homeomorphism?

We conclude this collection of informal questions and remarks with a bounded version of the Borel conjecture:

Question 6.5. Suppose that $E \Gamma_{0}$ is a topological manifold. Is every bounded homotopy equivalence $W \rightarrow E \Gamma_{0}$, bounded with respect to the identity map $E \Gamma_{0} \rightarrow$ $E \Gamma_{0}$, boundedly homotopic to a homeomorphism ?

We remark that if the bounded Borel conjecture is true for a torsion-free group $\Gamma_{0}$, then the integral $L$-theory assembly map for $\Gamma_{0}$ is a split monomorphism of spectra [2], [10]. This is a strong version of the Novikov conjecture. 


\section{REFERENCES}

[1] Bestvina, M., and Mess, G., The boundary of hyperbolic groups, Journal of the AMS 4 (1991), 469-481.

[2] Carlsson, G. and Pedersen, E. K., Controlled algebra and the Novikov conjectures for K- and L-theory, Topology 34 (1995), 731-758.

[3] Ferry, S. C., and Pedersen, E. K., Epsilon surgery theory, Novikov Conjectures, Rigidity and Index Theorems, Vol. 2 (Oberwolfach, 1993), London Math. Soc. Lect. Notes 227, Cambridge University Press, Cambridge, 1995, pp. 167-226.

[4] Gromov, M., Asymptotic invariants of infinite groups, Geometric Group Theory, Vol. 2 (Sussex, 1991), London Math. Soc. Lect. Notes 182, Cambridge University Press, Cambridge, 1993, pp. 1-295.

[5] Hambleton, I. and Pedersen, E. K., Non-linear similarity revisted, Prospects in Topology (ed. F. Quinn), Annals of Math. Studies 138, Princeton University Press, Princeton, NJ, 1995, pp. 157-174.

[6] Hambleton, I. and Pedersen, E. K., Bounded surgery and dihedral group actions on spheres, Journal of the AMS 4 (1991), 105-126.

[7] Higson, N., Pedersen, E. K., and Roe, J., $C^{*}$-algebras and controlled topology, K-Theory 11 (1997), 209-239.

[8] Kister, J. M., Differentiable periodic actions on $E^{8}$ without fixed points, Amer. Math. J. 85 (1963), 316-319.

[9] Martin, G. J., Infinite group actions on spheres, Revista Matemática Iberoamericana 4 (1988), 407-451.

[10] Pedersen, E. K., Bounded and continuous control, Novikov Conjectures, Rigidity and Index Theorems, Vol. 2 (Oberwolfach, 1993), London Math. Soc. Lect. Notes 227, Cambridge University Press, Cambridge, 1995, pp. 277-284.

Department of Mathematics \& Statistics, McMaster University, Hamilton, Ont. L8S $4 \mathrm{~K} 1$, CANADA

E-mail address: ian@math.mcmaster.ca

Department of Math. Sci., Binghamton University, Binghamton, Ny 13902-6000, USA

E-mail address: erik@math.binghamton.edu 